\title{
A Note on Chambers' Method for Finding a Zero of a Function
}

\author{
By M. G. Cox
}

\begin{abstract}
A class of methods due to Chambers is compared with the secant method and the method of inverse quadratic interpolation.
\end{abstract}

Recently, Chambers [1] has described a class of iterative methods for finding a simple isolated zero of a function $f(x)$ which purport to have quadratic or superquadratic convergence exponents. He compares his methods with a method based upon inverse quadratic interpolation, which possesses a convergence exponent of 1.839. The $n$th iteration of the latter method consists of interpolating inversely the function values $f_{n-2}, f_{n-1}$ and $f_{n}$, which correspond to the latest three estimates $x_{n-2}$, $x_{n-1}$ and $x_{n}$ of the zero, by means of a quadratic, in order to obtain a new estimate $x_{n+1}$. Thus, denoting the iteration function for this method by $\phi$,

$$
x_{n+1}=\phi\left(x_{n-2}, x_{n-1}, x_{n}\right) .
$$

The value of $f_{n+1}$ is then computed and the $(n+1)$ th iteration started.

Chambers' methods use the modified formula

$$
x_{n+1}=\phi\left(x_{n-1}, x_{n}, x_{n}^{*}\right),
$$

where $x_{n}^{*}$ is some function of $x_{n-1}$ and $x_{n}$. He shows that various definitions of $x_{n}^{*}$ give rise to convergence exponents of 2, 2.414 and 2.732. However, each application of the iteration function (2) requires two evaluations of the function $f$, viz. $f_{n}$ and $f_{n}^{*}$, as opposed to (1) which requires just one. It follows that for a fairer comparison with methods which require just one function evaluation per iteration the square roots of the above three convergence exponents should be taken. Note that an iteration function constructed by composition of the secant method with itself [2] also requires just two function evaluations per iteration and has a convergence exponent of $(1.618)^{2}=2.618$. Furthermore, composition of the iteration function (1) with itself, yielding

$$
x_{n+1}=\phi\left(x_{n-2}, x_{n-1}, \phi\left(x_{n-3}, x_{n-2}, x_{n-1}\right)\right),
$$

again uses the same amount of information, but possesses a convergence exponent of $(1.839)^{2}=3.382$, which is superior to all those derived by Chambers.

Received August 17, 1971.

AMS 1970 subject classifications. Primary 65H05.

Key words and phrases. Approximation to the root of an equation.

Copyright @ 1972, American Mathematical Society 
Division of Numerical Analysis \& Computing National Physical Laboratory

Middlesex, England

1. Ll. G. Chambers, "A quadratic formula for finding the root of an equation," Math. Comp., v. 25, 1971, pp. 305-307.

2. J. F. Traub, Iterative Methods for the Solution of Equations, Prentice-Hall Series in Automatic Computation, Prentice-Hall, Englewood Cliffs, N.J., 1964, p. 185. MR 29 \#6607. 